\title{
The locus of temporal preparation effects: Evidence from the psychological refractory period paradigm
}

\author{
KARIN M. BAUSENHART and BETTINA ROLKE \\ University of Tübingen, Tübingen, Germany \\ STEVEN A. HACKLEY \\ University of Missouri, Columbia, Missouri \\ and \\ ROLF ULRICH \\ University of Tübingen, Tübingen, Germany
}

\begin{abstract}
In reaction time (RT) tasks, responses are especially fast when participants can anticipate the onset of an imperative response signal. Although this RT facilitation is commonly attributed to temporal preparation, it is unclear whether this preparation shortens the duration of early or late processes. We used the effect propagation property of the psychological refractory period paradigm to localize the effect of temporal preparation. Manipulation of temporal uncertainty affected the RT of Task 1, regardless of the level of stimulus onset asynchrony (SOA). Consistent with the prediction of an early locus of temporal preparation, this effect propagated completely to the RT of Task 2 at short SOAs, but propagation diminished virtually to zero at long SOAs.
\end{abstract}

When participants are required to react quickly to a response signal, a considerable reduction of RT is usually observed when temporal uncertainty about its occurrence is minimized. Since the classical study of Woodrow (1914), this phenomenon has been repeatedly demonstrated (Niemi \& Näätänen, 1981) and shown to be robust; for example, it is not only observed for simple RTs, but also for choice RTs (see, e.g., Simon \& Slaviero, 1975). The effect has been studied by manipulating the foreperiod duration between a warning signal and the imperative response signal. According to Niemi and Näätänen, the warning signal reduces the uncertainty about when the response signal will occur, which in turn promotes the temporal preparation of the response.

An unresolved issue of theoretical importance, however, concerns the locus of temporal preparation within the processing stream from input to the corresponding response (for reviews, see Hackley \& Valle-Inclán, 2003; Müller-Gethmann, Ulrich, \& Rinkenauer, 2003). Some theorists assume that temporal preparation enhances the speed of late motor processes (e.g., Sanders, 1980, 1998). According to this prevailing view, the reduction of tem-

We thank Charles Folk, Hal Pashler, Hannes Schröter, and Jeff Miller for helpful comments, and the University of Tübingen for supporting a sabbatical stay for Steven Hackley at our department. Correspondence should be sent to K. Bausenhart or B. Rolke, Psychologisches Institut, Universität Tübingen, Friedrichstr. 21, 72072 Tübingen, Germany (e-mail: karin.bausenhart@uni-tuebingen.de or bettina.rolke@uni-tuebingen.de). poral uncertainty shortens the late motoric portion of RT. Other theorists, however, hold that temporal preparation operates at an earlier level; they assume that the reduction of temporal uncertainty speeds up perceptual processing or response selection (e.g., Hackley \& Valle-Inclán, 2003; Müller-Gethmann et al., 2003; Posner, 1978).

A review of the relevant studies provided a somewhat mixed picture (Müller-Gethmann et al., 2003). Several studies employing the additive-factors method (Sternberg, 2001) have concluded that a manipulation of temporal uncertainty changes the speed of motor processes. This conclusion has been supported by several other studies that assessed foreperiod effects on measures other than RT, such as response force, reflex amplitude, transcranially evoked motor potentials, hemodynamic imaging, and the contingent negative variation.

Recent chronopsychophysiological studies, however, have challenged the notion that temporal preparation operates exclusively or primarily at a late motoric level (Hackley \& Valle-Inclán, 1998, 1999; Müller-Gethmann et al., 2003; Smulders, 1993). These studies employed the lateralized readiness potential (LRP) to bisect RT into early and late phases. Contrary to the prevailing view, the results of these studies have shown that a reduction of temporal uncertainty shortens the early but not the late portion of RT. Hence, these studies support the conclusion that temporal preparation facilitates the speed of early processes. As noted above, response force, neuroimaging, and other amplitude measures provide indisputable evidence that temporal preparation does influence late motor processes. 
What the LRP data indicate is simply that temporal preparation effects on the speed of response initiation are due to some other, earlier mechanism.

In the study reported below, we used the psychological refractory period (PRP) paradigm to obtain further and independent evidence regarding the locus of temporal preparation effects on the speed of response initiation (see Pashler \& Johnston, 1998, for a review of this paradigm). This paradigm was originally developed to resolve the temporal microstructure of dual-task interference, but more recently it has also been employed to localize several experimental effects (see, e.g., Miller \& Reynolds, 2003; Ruthruff, Johnston, \& Van Selst, 2001).

This paradigm requires that participants perform two successive yet temporally overlapping RT tasks. For example, in the present experiment, the first response signal (S1) required color discrimination: Specifically, a red or green response signal appeared on the monitor, and depending on the color, participants were to press a key with the middle or index finger of the left hand. The second response signal (S2) was a high-, medium-, or low-pitched tone that appeared shortly after $\mathrm{S} 1$. The participants were to respond to this signal with the other hand, indicating with the index finger that the tone was low, with the middle finger that it was medium, and with the ring finger that it was high.

The standard PRP finding is that the reaction time for S2 (RT2) increases dramatically as the SOA between S1 and $\mathrm{S} 2$ decreases. Although various accounts of this PRP effect have been proposed (e.g., Meyer \& Kieras, 1997; Navon \& Miller, 2002; Tombu \& Jolicœur, 2003), there is strong evidence that the effect emerges from a response selection bottleneck (e.g., Pashler, 1994; Sommer, Leuthold, \& Schubert, 2001). Accordingly, response selection for $S 2$ cannot begin until the response selection process for $\mathrm{S} 1$ has finished. Specifically, this bottleneck model assumes that response selection constitutes a single-channel process that cannot serve both tasks simultaneously. This central processor receives its input from perceptual processes and forwards its output to motor processes. These pre- and post-bottleneck processes proceed without interference from the other task.

The bottleneck model involves an effect propagation property (see Miller \& Reynolds, 2003) that we employed as a tool to assess the locus of temporal preparation (Figure 1). ${ }^{1}$ First note that at short SOAs, the perceptual outcome resulting from $\mathrm{S} 2$ processing cannot be fed into the central processor (the bottleneck) until this processor has selected the first response and forwarded this information to the motor system. Crucially, at short SOAs, any experimental manipulation (e.g., of temporal uncertainty) that affects the duration of Task 1 perceptual processing or response selection will be propagated to Task 2 processing and will affect RT2 accordingly.

For example, suppose an experimental manipulation prolongs perceptual processing of Task 1 by $50 \mathrm{msec}$ (Figure 1B). In this case, the initiation of the central bottleneck process by Task 1 would also be delayed by $50 \mathrm{msec}$, and RT2 would consequently be prolonged by this amount of

\section{A. Low Temporal Uncertainty}

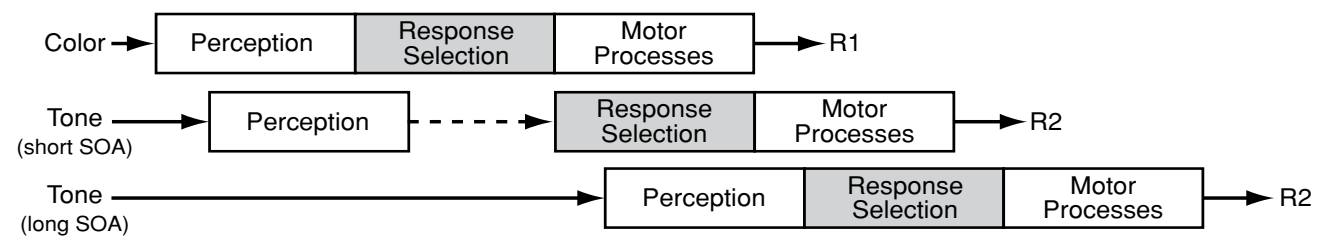

\section{B. High Temporal Uncertainty}

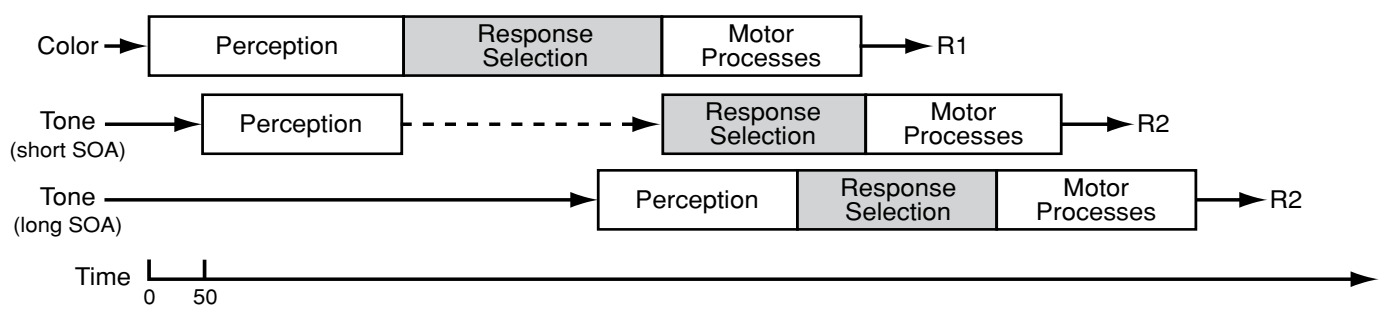

Figure 1. Illustration of effect propagation. This figure depicts dual-task processing in accordance with the bottleneck model for trials with short and long SOAs and for trials with low (A) and high (B) temporal uncertainty. (A) The upper sequence illustrates the duration of perceptual processing, response selection, and motor processing for Task 1. The durations of these stages do not depend on the SOA. The middle sequence shows the same processing sequence for Task 2 with a short SOA. Note that response selection for Task 2 cannot begin until response selection for Task 1 has finished; this waiting period is represented by the dashed line. The third sequence depicts Task 2 processing with a long SOA. Here response selection for Task 2 can immediately begin after Task 2 perception has finished. (B) The lower sequence is identical to the one in panel $A$ except that, due to high temporal uncertainty, longer premotor processing emerges in Task 1. Comparison of panels $A$ and $B$ illustrates that an increase in RT1 associated with higher temporal uncertainty would completely propagate to RT2 at a short SOA. At a long SOA, however, this effect on RT1 would no longer be propagated to RT2. 
time. At long SOAs, however, Task 2 need not wait until the central processor is finished with Task 1 processing, and effect propagation should no longer occur. Therefore, if temporal preparation affects early processes of Task 1 , effect propagation on RT2 should be observed at short but not at long SOAs. If, however, temporal preparation operates on late processes, no such effect propagation should be observed for RT2 at either short or long SOAs.

\section{METHOD}

One third of the participants performed in the above-mentioned dual-task condition. This condition assessed whether or not a manipulation of temporal uncertainty on S1 produces an effect propagation to RT2. Another third of the participants were instructed to ignore $\mathrm{S} 2$ and to perform Task 1 only (visual single-task condition). This single-task condition served as a control condition to determine the size of the foreperiod effect and to assess potential accessory effects (Hackley \& Valle-Inclán, 1998, 1999) of S2 on RT1, which could hamper the interpretation of the results in the dual-task condition. The third group of participants received the same stimuli as the other two groups, but they ignored S1 and performed Task 2 only (auditory single-task condition). The purpose of this single-task condition was to determine possible direct effects of the warning signal on Task 2 , thereby providing a baseline against which propagation effects in the dual-task condition could be assessed. It was anticipated that direct effects of foreperiod manipulation would be modest in size, on the assumption that S1 would serve as a more useful predictor of S2 onset than would the earlier warning signal.

\section{Participants}

Forty-seven women and 7 men participated (mean age $=$ 24.3 years). According to self-report, all but 3 were right-handed, and all had normal or corrected-to-normal vision.

\section{Stimuli and Apparatus}

An IBM-compatible computer presented stimuli and recorded both responses and RTs. All visual stimuli were presented in the center of the screen on a blue background $\left(2.5 \mathrm{~cd} / \mathrm{m}^{2}\right)$ and viewed from a distance of approximately $50 \mathrm{~cm}$. The fixation cross $(2 \times$ $2 \mathrm{~mm}, 110 \mathrm{~cd} / \mathrm{m}^{2}$ ) and the warning signal (an asterisk with a diameter of $9 \mathrm{~mm}, 110 \mathrm{~cd} / \mathrm{m}^{2}$ ) were white. S1 was a filled red or green rectangle $\left(6 \times 9 \mathrm{~mm}, 25\right.$ and $70 \mathrm{~cd} / \mathrm{m}^{2}$, respectively). S2 was a low-, medium-, or high-pitched tone $(800,1000$, or $1200 \mathrm{~Hz})$ presented binaurally over headphones at approximately $70 \mathrm{~dB}(\mathrm{~A})$. There were separate response panels for each hand with response buttons for each finger. Two buttons on one panel were for the middle and index fingers of the left hand (Task 1), and three response buttons on the other panel were assigned to the index, middle, and ring fingers of the right hand (Task 2).

\section{Procedure}

Each intertrial interval started with the presentation of the fixation cross, which was displayed for an interval of $(1.0+x)$ sec, with $x$ a random variable that followed an exponential distribution with a mean of $2 \mathrm{sec}$. This random interval was introduced to emphasize the importance of the warning signal as a temporal reference for preparation (Müller-Gethmann et al., 2003). At the end of the intertrial interval, the fixation cross was replaced by the warning signal (asterisk) for $200 \mathrm{msec}$. At warning signal offset, the fixation cross reappeared and was again replaced after $600,1,400$, or $3,000 \mathrm{msec}$ by $\mathrm{S} 1$. Thus, the foreperiod length between the onset of the warning signal and $\mathrm{S} 1$ was either $800,1,600$, or $3,200 \mathrm{msec}$ for any given block of trials. S1 remained on the screen for $200 \mathrm{msec}$ and was then replaced by the fixation cross, which stayed on until the participant had responded to either S1 or S2 (single-task conditions) or to both (dual-task condition), at which point the trial ended. At a variable SOA of 50, 200, or $800 \mathrm{msec}$ following S1 onset, S2 sounded for $200 \mathrm{msec}$. In case of an incorrect response, the word FEHLER (error) appeared in the lower part of the screen for $500 \mathrm{msec}$ at the end of the trial.

A single session lasted about $1.5 \mathrm{~h}$ and consisted of 15 blocks. Foreperiod length was kept constant over five consecutive blocks of 36 trials each. Order of the three foreperiod conditions was balanced across participants in each group. The first block of each foreperiod condition was considered practice and was discarded from data analysis. After each block, the participants received performance feedback concerning mean RT and percentage of errors.

\section{Design}

For the single- and dual-task conditions, the experiment factorially combined foreperiod length $(800,1,600$, and 3,200 $\mathrm{msec})$ and SOA $(50,200$, and $800 \mathrm{msec})$. This design yielded a total of 48 experimental trials per participant for each factorial combination of experimental conditions.

\section{RESULTS}

RTs shorter than 150 or greater than $1,500 \mathrm{msec}$ were considered outliers, and their corresponding trials were discarded. Specifically, $0.3 \%$ of all trials were eliminated in the visual single-task condition, $0.3 \%$ in the auditory single-task condition, and $4.8 \%$ in the dual-task condition (RT1, 1.2\%; RT2, 4.6\%). Separate repeated measures ANOVAs with the factors foreperiod and SOA were conducted on mean RTs and percentages of correct responses (PCs). Parallel analyses in which fast and slow outliers were not omitted yielded results similar to those reported below. In all analyses, $p$ values were adjusted, whenever appropriate, for violations of the sphericity assumption using the Greenhouse-Geisser correction.

\section{Single-Task Conditions}

The results for the single-task conditions are summarized in Figure 2, which depicts mean RTs and PCs for both conditions as a function of foreperiod length and SOA. An ANOVA on PC for the visual single-task condition revealed only a main effect of SOA $[F(2,34)=9.6$, $p=.002]$ due to more incorrect responses at the shortest SOA. No further significant results were obtained (all $p \mathrm{~s}>$.162). This single-task condition revealed that the manipulation of temporal uncertainty was successful, because foreperiod length produced a strong effect on RT $[F(2,34)=45.2, p<.001]$. As one would expect, mean RT increased with foreperiod length, apparently as a result of increasing temporal uncertainty. Mean RT increased slightly yet significantly with SOA $[F(2,34)=4.8, p=$ .017]. Crucially, the two factors produced no significant interaction on RT $(F<1)$, indicating that the taskirrelevant S2 did not modulate the observed effect of temporal uncertainty in Task 1.

An ANOVA on PC for the auditory single-task condition revealed no significant effects $(F \mathbf{s}<1)$. An ANOVA on RT revealed no significant effect of SOA $(F<1)$, and as expected, the direct effect on RT of foreperiod length was modest in size $[F(2,34)=5.07, p=.034]$. Presumably, the temporally proximal S1 (SOAs of $50-800 \mathrm{msec}$ ) constituted a more useful warning signal than did the more 


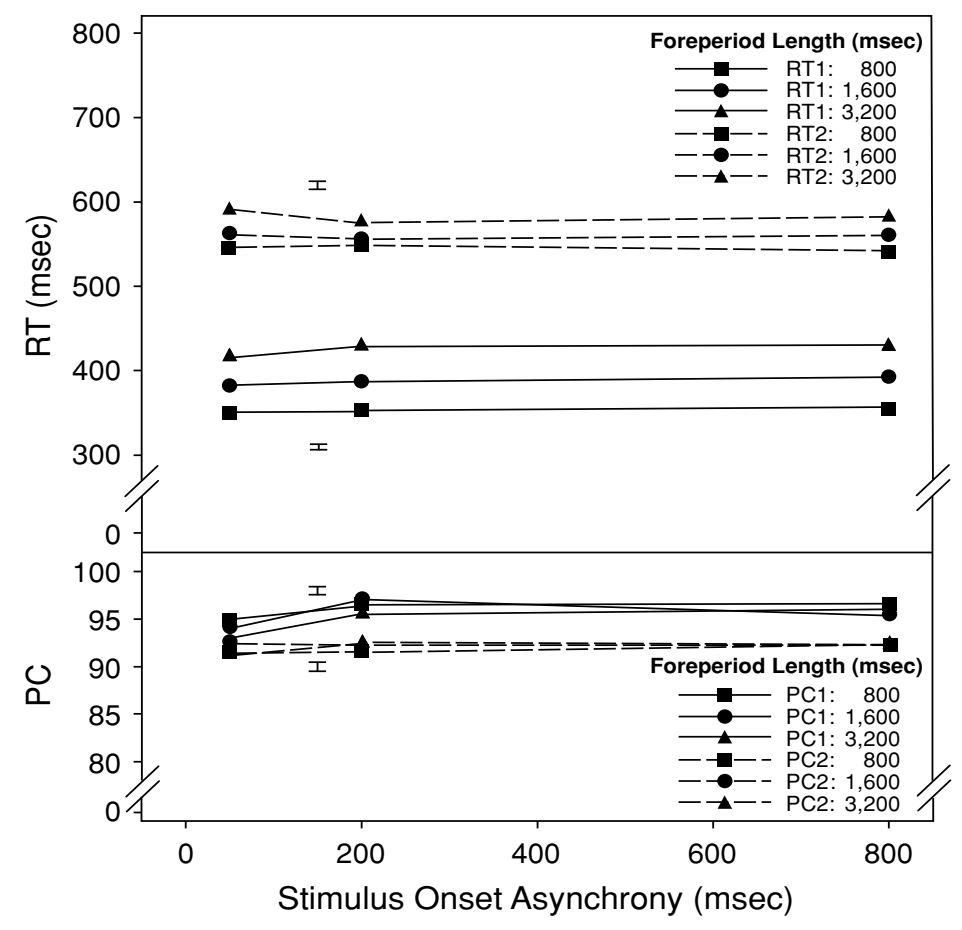

Figure 2. Results for the single-task conditions. (Top) Mean reaction times (RT) for the visual (RT1) and auditory (RT2) single-task conditions as a function of foreperiod length and SOA. (Bottom) Percentages of correct responses (PC) for the visual (PC1) and auditory (PC2) singletask conditions as a function of foreperiod length and SOA. The error bars in each panel provide standard errors of the mean. These standard errors were computed from the pooled error terms of the corresponding ANOVA according to a suggestion made by Loftus (2002).

distal asterisk (SOAs of $850-4,000 \mathrm{msec}$ ). This assumption is consistent with previous research showing that the presence of a proximal warning signal can eliminate the foreperiod effect of an earlier warning signal (Low, Larson, Burke, \& Hackley, 1996). Most importantly, and analogous to the visual single-task condition, SOA and foreperiod did not interact $(F<1)$. These findings imply that, should an SOA $\times$ foreperiod interaction be observed in the dual-task condition, it could reasonably be attributed to effect propagation.

\section{Dual-Task Condition}

Figure 3 summarizes the results for the dual-task condition. The upper panel represents mean RTs for each task, and the lower panel PCs. There was no effect on PC (all $p$ s $>$.144). All trials with interresponse intervals less than $100 \mathrm{msec}$ were defined as grouped responses, which can undermine the interpretation of PRP data, and were thus eliminated from RT analyses. ${ }^{2}$

An ANOVA on RT1 revealed again a strong effect of foreperiod length $[F(2,34)=8.2, p=.001]$, confirming that the single-task foreperiod effect generalized to the dual-task situation. However, in contrast with the singletask conditions, the effect saturated faster in the dual-task condition, most likely because of increased task difficulty. It is known that estimating time intervals places high de- mands on resources (Brown, 1997), as does maintaining response readiness (Gottsdanker, 1975). Assuming that these processes are more difficult for longer foreperiods (Niemi \& Näätänen, 1981; Requin, Brener, \& Ring, 1991), dual-task interference might be expected to yield an earlier asymptote for foreperiod effects. Also, RT1 decreased with increasing SOA $[F(2,34)=14.9, p<$ $.001]$. This presumably happened because processing of Task 1 was interrupted by Task 2 during some trials with short SOAs. The interaction of foreperiod and SOA was not significant for RT1 $[F(4,68)=1.1, p=.367]$.

In the analogous ANOVA on RT2, the usual PRP effect was obtained; that is, RT2 increased substantially as SOA decreased $[F(2,34)=203.3, p<.001]$. Theoretically more important, however, was the finding that RT2 increased with foreperiod length $[F(2,34)=7.4, p=$ $.003]$. In addition, and as predicted by the effect propagation account, this effect decreased virtually to zero as SOA increased $[F(4,68)=5.6, p=.003]$; in fact, a post hoc analysis revealed no effect at the longest SOA $(p=.662)$. At the two shortest SOAs, the size of the foreperiod effect on RT1 was fully propagated to RT2. This was confirmed statistically by computing the difference between the long $(3,200-\mathrm{msec})$ and short $(800-\mathrm{msec})$ foreperiods separately for RT1 and RT2 and then comparing these two differences. In the 50-msec-SOA condition, the foreperiod ef- 


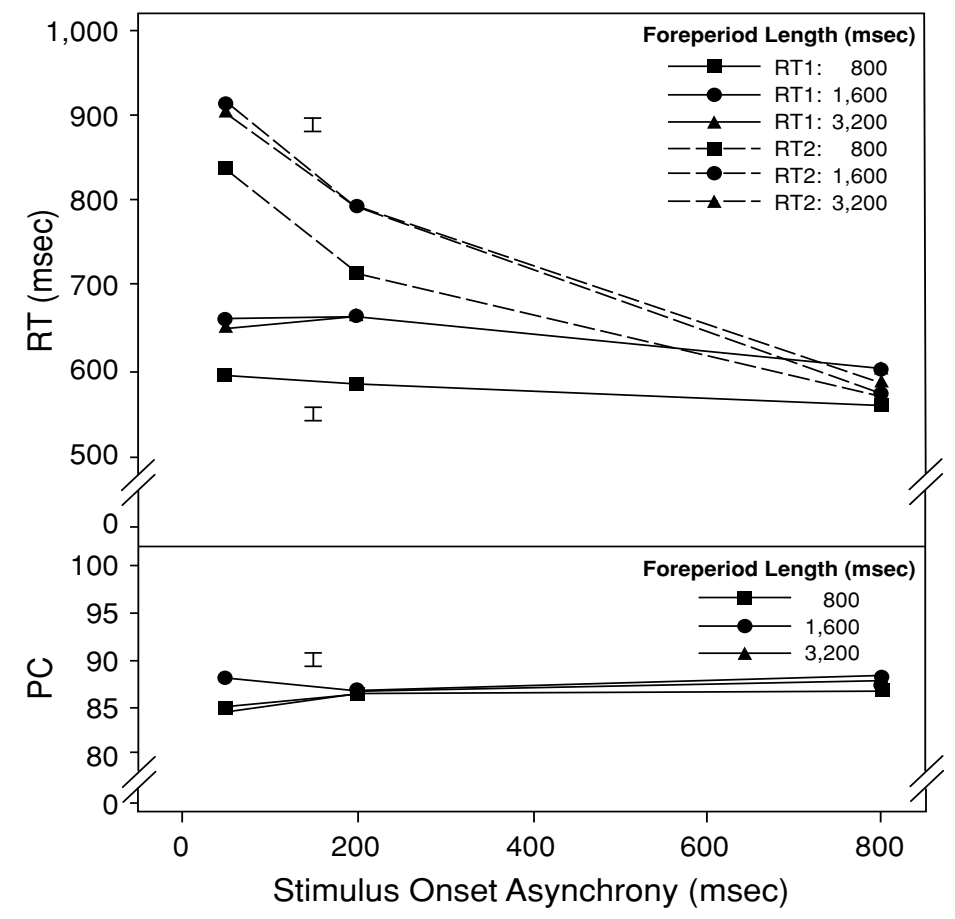

Figure 3. Results for the dual-task condition. (Top) Mean reaction times (RT1 and RT2) as a function of foreperiod length and SOA. (Bottom) Percentages of correct responses (PC) as a function of foreperiod length and SOA.

fect was $55 \mathrm{msec}$ for RT1 and $64 \mathrm{msec}$ for RT2, which did not differ statistically $(p=.479)$. In the 200 -msec-SOA condition, the corresponding values were virtually identical ( $77 \mathrm{vs} .78 \mathrm{msec})$.

As noted earlier, the auditory single-task control condition showed that any direct effect of foreperiod length on RT2 was negligible, presumably because S1 constituted a more useful warning signal than did the asterisk. To provide further evidence that foreperiod effects on RT2 in the dual-task condition were primarily due to effect propagation, we compared the sizes of the foreperiod effects on RT2 in the auditory single-task and the dual-task conditions. Under the effect propagation hypothesis, foreperiod effects should be larger in the dual-task than in a singletask condition when the SOA is short. This prediction was confirmed by a significant foreperiod $\times$ SOA $\times$ task interaction $[F(4,136)=5.52, p<.001]$.

\section{DISCUSSION}

This study was conducted in order to localize the effect of temporal preparation in the processing chain from input to response. Specifically, we addressed the question of whether temporal preparation enhances the speed of motor or premotor processes. The effect propagation property of bottleneck models was used as an inferential tool to answer this question. The present results are clear cut. First, and as expected, a sizeable foreperiod effect on RT1 was observed in the visual single-task condition. Sec- ond, essentially the same effect on RT1 was obtained in the dual-task condition at all SOAs. Third, and crucially, this foreperiod effect on RT1 carried over at full size to RT2 at short SOAs, and yet was virtually nil at the longest SOA. As explained in the introduction, this pattern of results supports the assumption that temporal preparation exerts its effect prior to the completion of response selection.

The finding that the effect on RT2 disappears at long SOAs is also important for ruling out an alternative interpretation of our results. According to this alternative view, foreperiod effects for RT2 do not reflect effect propagation from Task 1 but instead direct effects of the warning signal on Task 2. This view emphasizes that the asterisk did not just reduce temporal uncertainty for the onset of S1 but also for the onset of S2. It does appear likely that the asterisk helped the dual-task participants to prepare Task 2, given the significant (albeit small) foreperiod effect in the auditory single-task condition. However, if this were the basis for foreperiod effects on the dual-task RT2, there would be no reason to expect the foreperiod effect to be larger in the dual-task condition or to disappear at the longest SOA. Such a pattern is predicted under the effect propagation hypothesis, and this is indeed what was observed.

Thus, this study provides evidence for the notion (Posner, 1978) that temporal preparation enhances the speed of premotor processes but does not change the speed of motor processes. This conclusion completely agrees with the results from recent chronophysiological studies in which temporal preparation was manipulated using 
short foreperiods (Hackley \& Valle-Inclán, 1998, 1999); short, medium, and long foreperiods (Müller-Gethmann et al., 2003); and variable versus fixed intertrial intervals (Smulders, 1993). For example, in Müller-Gethmann et al.'s study, foreperiod length varied between 50 and $6,400 \mathrm{msec}$ in different blocks of trials but was kept constant within blocks of trials. As in the present study, the imperative response signal demanded a speeded choice response. Responses were fastest at foreperiods of around $300 \mathrm{msec}$ and slowed down with increasing foreperiod length. Müller-Gethmann et al. examined the locus of the effect of temporal preparation using the onset of the lateralized readiness potential as a landmark to partition RT into two segments, one including perceptual, decision, and early motor stages, and the other only late motoric processes. More specifically, they tested the hypothesis that temporal preparation influences the speed of late motoric processes.

This hypothesis was proposed by Sanders (1980) based on behavioral data generated within an additivefactors framework. Relevant data included the finding that instructed muscle tension modulates the foreperiod effect on RT, but stimulus clarity does not (Sanders, 1980, 1998). Interestingly, and contrary to the prediction of this hypothesis, the length of the LRP-to-keypress interval, which indexes the duration of late motoric processes, was not systematically influenced by foreperiod length. By contrast, variations in the duration of the stimulus-toLRP interval resembled almost perfectly the foreperiod effect on RT. On the basis of this rather unexpected result, Müller-Gethmann et al. (2003) concluded that these LRP findings disagree with the prevailing view, according to which temporal preparation affects late motor stages.

Our conclusion of an early effect does not deny the possibility that temporal preparation affects various aspects of response preparation and execution other than speed. For example, it has been demonstrated that the force of a response is sensitive to a manipulation of temporal uncertainty (Mattes \& Ulrich, 1997); participants produce more force when temporal uncertainty is high, and this finding clearly suggests that temporal preparation operates at a motor level. Nevertheless, it has been repeatedly reported that RT and the dynamics of the response are uncorrelated (see, e.g., Mordkoff, Miller, \& Roch, 1996). This lack of a correlation is inconsistent with the idea that changes in response dynamics are accompanied by changes in the duration of motor or premotor processes. Thus, motor adjustments that accompany temporal preparation may serve a different purpose than speeding up responses. Analogous arguments may be applied to other physiological markers of motor preparation, such as regional cerebral blood oxygenation levels and modulation of probe reflex amplitudes.

One may argue that the logic of this effect propagation approach, and therefore the present conclusion, depends greatly on the validity of bottleneck models. The basic premise of this model class is the existence of a central processor that can only operate on one task at a time. For example, capacity models reject such a structural bottleneck and assume instead that both tasks need to share limited resources (Tombu \& Jolicœur, 2003). Because this alternative model class implies the same effect propagation property as structural bottleneck models (Navon \& Miller, 2002), however, our conclusions may be robust with respect to violation of this key assumption.

In conclusion, this study demonstrates once again the usefulness of the PRP paradigm in identifying the stages of response processing that are affected by experimental manipulations. In contrast to the prevailing notion that temporal preparation operates exclusively on late motor processes, the present study provides further evidence for an earlier locus of temporal preparation.

\section{REFERENCES}

Brown, S. W. (1997). Attentional resources in timing: Interference effects in concurrent temporal and nontemporal working memory tasks. Perception \& Psychophysics, 59, 1118-1140.

GOTTSDANKER, R. (1975). The attaining and maintaining of preparation. In P. M. A. Rabbitt \& S. Dornic (Eds.), Attention and performance $V$ (pp. 33-49). New York: Academic Press.

Hackley, S. A., \& Valle-Inclán, F. (1998). Automatic alerting does not speed up late motoric processes in a reaction-time task. Nature, 391, 786-788.

HaCKLEy, S. A., \& VAlle-InClán, F. (1999). Accessory stimulus effects on response selection: Does arousal speed decision making? Journal of Cognitive Neuroscience, 11, 321-329.

HaCKley, S. A., \& VAlle-Inclán, F. (2003). Which stages of processing are speeded by a warning signal? Biological Psychology, 64, 27-45.

LofTus, G. R. (2002). Analysis, interpretation, and visual presentation of experimental data. In H. Pashler (Ed.), Stevens' handbook of experimental psychology: Vol. 4. Methodology in experimental psychology (3rd ed., pp. 339-390). New York: Wiley.

Low, K. A., Larson, S. L., Burke, J., \& Hackley, S. A. (1996). Alerting effects on choice reaction time and the photic blink reflex. Electroencephalography \& Clinical Neurophysiology, 98, 385-393.

Mattes, S., \& Ulrich, R. (1997). Response force is sensitive to the temporal uncertainty of response stimuli. Perception \& Psychophysics, 59, 1089-1097.

Meyer, D. E., \& Kieras, D. E. (1997). A computational theory of executive cognitive processes and multiple-task performance: II. Accounts of psychological refractory-period phenomena. Psychological Review, 104, 749-791.

Miller, J., \& ReYNOLDS, A. (2003). The locus of redundant-targets and nontargets effects: Evidence from the psychological refractory period paradigm. Journal of Experimental Psychology: Human Perception \& Performance, 29, 1126-1142.

Mordkoff, J. T., Miller, J., \& Roch, A. C. (1996). Absence of coactivation in the motor component: Evidence from psychophysiological measures of target detection. Journal of Experimental Psychology: Human Perception \& Performance, 22, 25-41.

Müller-Gethmann, H., Ulrich, R., \& Rinkenauer, G. (2003). Locus of the effect of temporal preparation: Evidence from the lateralized readiness potential. Psychophysiology, 40, 597-611.

Navon, D., \& Miller, J. (2002). Queuing or sharing? A critical evaluation of the single-bottleneck notion. Cognitive Psychology, 44, 193-251.

NiEMI, P., \& N̈̈̈̈TÄNEN, R. (1981). Foreperiod and simple reaction time. Psychological Bulletin, 89, 133-162.

PAshler, H. (1994). Dual-task interference in simple tasks: Data and theory. Psychological Bulletin, 116, 220-244.

Pashler, H., \& Johnston, J. C. (1998). Attentional limitations in dualtask performance. In H. Pashler (Ed.), Attention (pp. 155-189). Hove, U.K.: Psychology Press.

Posner, M. I. (1978). Chronometric explorations of mind. Hillsdale, NJ: Erlbaum.

Requin, J., Brener, J., \& Ring, C. (1991). Preparation for action. In J. R. Jennings \& M. G. H. Coles (Eds.), Handbook of cognitive psychophysiology: Central and autonomic nervous system approaches (pp. 357-448). Chichester, U.K.: Wiley. 
Ruthruff, E. D., Johnston, J. C., \& VAn Selst, M. (2001). Why practice reduces dual-task interference. Journal of Experimental Psychology: Human Perception \& Performance, 27, 3-21.

SANDERS, A. F. (1980). Some effects of instructed muscle tension on choice reaction time and movement time. In R. S. Nickerson (Ed.), Attention and performance VIII (pp. 59-74). Hillsdale, NJ: Erlbaum.

SANDERS, A. F. (1998). Elements of human performance: Reaction processes and attention in human skill. Mahwah, NJ: Erlbaum.

Simon, R. J., \& Slaviero, D. P. (1975). Differential effects of a foreperiod countdown procedure on simple and choice reaction time. Journal of Motor Behavior, 7, 9-14.

SMULDERS, F. T. Y. (1993). The selectivity of age effects on information processing: Response times and electrophysiology. Unpublished doctoral dissertation, University of Amsterdam.

Sommer, W., Leuthold, H., \& Schubert, T. (2001). Multiple bottlenecks in information processing? An electrophysiological examination. Psychonomic Bulletin \& Review, 8, 81-88.

SternberG, S. (2001). Separate modifiability, mental modules, and the use of pure and composite measures to reveal them. Acta Psychologica, 106, 147-246.
ToMbu, M., \& Jolicceur, P. (2003). A central capacity sharing model of dual-task performance. Journal of Experimental Psychology: Human Perception \& Performance, 29, 3-18.

Woodrow, H. (1914). The effect upon reaction time of variation in the preparatory interval. In J. Angell, H. Warren, J. Watson, \& S. Franz (Eds.), The psychological monographs (pp. 16-65). Princeton, NJ: Psychological Review Company.

\section{NOTES}

1. The "effect propagation" paradigm is one of two methods by which PRP can be used to localize effects. An alternative, and more common, method is the "locus-of-slack" paradigm. See Miller and Reynolds (2003) for a lucid explanation of the two methods.

2 . Whether or not these trials were discarded had virtually no effect on the results of the RT1 and RT2 analyses.

(Manuscript received February 24, 2005; revision accepted for publication September 26, 2005.) 